\title{
Perfil ejecutivo de niños de 5 a 7 años en el cantón Ambato (Ecuador) Executive Profile in 5-to-7 Year-Old Children in Ambato (Ecuador)
}

\author{
Bel Fenellós, C. ${ }^{1}$, Flores Hernández, V.F. ${ }^{2}$, Del Rocio Tabares Rosero, X. ${ }^{2}$, Velastegui, R. ${ }^{2}$ \\ mbel@ucm.es, vf.flores@uta.edu.ec,rs.velastegui@uta.edu.ec,xdr.tabares@uta.edu.ec \\ .$^{1}$ Facultad de Educación \\ Universidad Complutense de Madrid \\ España, Madrid \\ ${ }^{2}$ Facultad de Ciencias Humanas y de la Educación \\ Universidad Técnica de Ambato \\ Ecuador, Ambato
}

\begin{abstract}
Resumen- Cada vez es mayor la importancia del estudio de las Funciones Ejecutivas, tanto los aspectos evolutivos como su relevancia en los procesos de aprendizaje. En este trabajo se presentan los resultados obtenidos por 135 estudiantes de Ambato, de 8 a 12 años, en la escala Atención-Función Ejecutiva de la batería de evaluación NEUROPSI. El análisis de los datos nos ha permitido comprobar que no existen diferencias significativas en función del sexo, y que los alumnos con puntuaciones extremas, por encima o por debajo del grupo normativo, son los que más años de escolaridad han cursado. La evaluación inicial de los alumnos de Ambato servirá de base para la realización de un programa de estimulación y fortalecimiento de su perfil ejecutivo.
\end{abstract}

Palabras clave: Funciones ejecutivas, Neuropsi atención y memoria, perfil ejecutivo.

\begin{abstract}
The importance of the study of Executive Functions, both the evolutionary aspects and their relevance in learning processes, is increasing. This paper presents the results obtained by 135 students from Ambato, between 8 to 12 years old, in the Attention-Executive Function scale of the NEUROPSI test battery. The analysis of the data has allowed us to verify that there are no significant differences according to sex and that the students with extreme scores, above or below the normative group, are those with more years of schooling. The initial test in students from Ambato will serve as a basis for the implementation of a program to stimulate and strengthen their executive profile. The initial test in students from Ambato will serve as a basis for the implementation of a program to stimulate and strengthen their executive profile.
\end{abstract}

Keywords: Executive functions, Neuropsi attention and memory, executive profile.

\section{INTRODUCCIÓN}

En 1982, Muriel Lezac utilizó el término Funciones Ejecutivas (FE) por primera vez. Posteriormente, las conceptualizó como

“... las capacidades que permiten a una persona funcionar con independencia, con un propósito determinado, con conductas autosuficientes y de manera satisfactoria...mientras las funciones ejecutivas permanezcan intactas, una persona puede sufrir pérdidas cognitivas considerables y continuar siendo independiente, constructivamente autosuficiente $y$ productiva. Cuando se alteran las funciones ejecutivas el sujeto no es capaz de autocuidarse, de realizar trabajos para otros, $n i$ de mantener relaciones sociales normales, independientemente de cómo conserve sus capacidades cognitivas" (Lezac, 1995, p 38).

La comunidad científica coincide con el autor en que las FE son el eje central que guía las conductas adaptativas y socialmente aceptadas y aceptables (Tirapu-Ustárroz, CorderoAndrés, Luna-Lario y Hernáez-Goñi, 2017).

Desde antes del nacimiento, el desarrollo neurobiológico del ser humano está íntimamente ligado con la adquisición de diferentes habilidades. La corteza prefrontal es considerada la estructura neuroanatómica base del funcionamiento ejecutivo. Ésta, contiene multitud de circuitos y estructuras particulares, y una jerarquía funcional especializada (García Molina, Enseñat, Tirapu-Ustárroz, y Roig-Rovira, 2009; Pino y Urrego, 2013; Soprano, 2003). El desarrollo de las FE es un proceso largo y complejo, que comienza en el embarazo y se extiende hasta la edad adulta. Está influido por gran variedad de procesos exógenos y endógenos (Anderson y Spencer-Smith, 2013). Las FE se desarrollan y mejoran rápidamente de los 3 a los 5 años, período en el que surgen las diferencias en las funciones cognitivas (Zelazo, Frye y Rapus, 1996). Además, la utilización de pruebas neuropsicológicas ha permitido examinar los déficits característicos asociados a condiciones específicas de la infancia, como el Trastorno por déficit de atención / hiperactividad (TDHA) (Curtis, Lindeke, Georgieff, y Nelson, 2002).

A pesar de la cada vez más prolífera investigación sobre el constructo, no existe consenso respecto a si nos encontramos ante un constructo unitario o un sistema de procesamiento multimodal con componentes independientes, pero interconectados (Tirapu et al., 2017). Lo que sí parece consensuado es que las FE representan la capacidad para adaptar nuestros recursos cognitivos en función de las demandas cambiantes del entorno (Gilbert y Burgess, 2008; Verdejo-García y Bechara, 2010).

Barkley $(1997,2001)$ propuso su modelo teórico enfatizando el papel de la conducta inhibitoria en el funcionamiento de las FE. Se basó en los aportes de Jacob Bronowski (1977) sobre el TDAH y el papel fundamental del lenguaje en la conducta humana, con su sustento en la corteza prefrontal. Integró, 
además, estos aportes iniciales con la teoría de las funciones prefrontales de Fuster (1997), el trabajo sobre memoria de trabajo de Goldman-Rakic (1995) y la hipótesis del marcador somático de Damasio (1996). La propuesta central del modelo de Barkley se basa en que la conducta inhibitoria favorece la autorregulación, así como la realización de acciones ejecutivas, ya que permite la demora en la decisión de responder.

Barkley (2001) define la conducta inhibitoria en función de tres procesos interrelacionados: la inhibición de una respuesta prepotente, la interrupción de una respuesta ya iniciada, y el control de interferencia. De acuerdo con esta propuesta, la inhibición conductual permite el correcto funcionamiento de otras cuatro FE: a) memoria de trabajo no verbal, b) memoria de trabajo verbal o internalización del lenguaje, c) autorregulación del afecto-motivación-arousal y d) reconstitución. Estas funciones son consideradas un sistema separado del de inhibición conductual, pero jerárquicamente organizadas. El modelo propone, además, que las FE maduran desde lo externo hacia lo interno; es decir, conceptualiza a las FE como formas de comportamiento autodirigido que evolucionan de respuestas manifiestas o públicas a respuestas encubiertas o privadas, como un medio para la autorregulación (Colombo, 2020).

En la última década, ha tenido una gran influencia el modelo propuesto por Miyake et al. (2000). En este modelo se consideran tres factores nucleares independientes: inhibición, memoria de trabajo y cambio. Es especialmente atractivo para la psicología del desarrollo, ya que valora estos componentes desde edades muy tempranas. El modelo excluye funciones consideradas comúnmente ejecutivas, tales como razonamiento, habilidad de planificación y organización.

También destaca el Sistema de Control Ejecutivo propuesto por Anderson $(2002,2008)$. Según este autor, las FE dependen de las funciones cognitivas de más alto nivel y de las de más bajo nivel, por lo que no se pueden considerar de forma aislada. No hay un consenso sobre las funciones que lo integran, pero si hay un acuerdo en considerar estas funciones especialmente importantes en la conducta cotidiana. Desde esta perspectiva, se categorizan las diferentes funciones ejecutivas en cuatro dominios interdependientes: procesamiento de la información, control atencional, flexibilidad cognitiva y establecimiento de objetivos. Estos cuatro dominios interaccionan y tienen relaciones bidireccionales. Anderson categoriza las diferentes medidas de las FE disponibles: a) control atencional (Attentional control); b) flexibilidad cognitiva (Cognitive flexibility); c) planteamiento de objetivos (Goal setting); y, d) procesamiento de la información (Information processing). Cada una de ellas encuentra detallada en la Tabla 1.

Tabla 1.

Categorías de las medidas de las FE.

multitareas y procesos de almacenamiento temporal (memoria de trabajo)

Planteamiento de objetivos (Goal setting) información

(Information processing)
Iniciativa, razonamiento conceptual, habilidad de planificación (anticipar futuros eventos, formulación de un objetivo, desarrollo de pasos para conseguir un objetivo) y organización (habilidad para organizar compleja información o secuenciar en fases el dominio de una estrategia de forma lógica y sistemática).

Se centra en la velocidad, fluencia y eficiencia para completar tareas nuevas o para resolver un problema.

\section{CONTEXTO}

La Universidad Técnica de Ambato planteó el Proyecto de Investigación "Potenciación de funciones ejecutivas a través de un programa de entrenamiento cerebral y aprendizaje mediado" con el objetivo principal de elaborar un programa de entrenamiento y potenciación de las $\mathrm{FE}$ en los escolares ecuatorianos.

Como primera tarea, se llevó a cabo la evaluación de 135 menores, cuyos resultados se muestran en este trabajo. De esta forma se ha establecido su perfil ejecutivo, sus fortalezas y debilidades. Estos datos han servido de base al diseño del programa de estimulación y refuerzo.

Estos datos nos han permitido el diseño de una batería de tareas, en realización, para la estimulación y potenciación de las FE.

\section{DESCRIPCIÓN}

La investigación realizada presenta un carácter clínico, descriptivo y transversal. La metodología de trabajo es cuantitativa, con la aplicación de la batería de evaluación y el análisis estadístico de los datos.

\subsection{Participantes}

La muestra estuvo compuesta por 135 estudiantes de nueve, diez, once y doce años de la ciudad de Ambato.

La participación de los menores fue voluntaria. Todos ellos presentaban de 5 a 7 años de estudio y pertenecían al cantón Ambato. Los estudiantes no presentaban rasgos de capacidades especiales.

Las pruebas de evaluación se aplicaron durante el tercer trimestre del 2020.

\begin{tabular}{|c|c|c|}
\hline Categoría & Definición & Las pruebas de evaluación se aplicaron durante el tercer \\
\hline $\begin{array}{l}\text { Control atencional } \\
\text { (Attentional control) }\end{array}$ & $\begin{array}{l}\text { Capacidad para atender selectivamente } \\
\text { a un estímulo específico }\end{array}$ & 3.2. Instrumento de recogida de datos \\
\hline $\begin{array}{l}\text { Flexibilidad cognitiva } \\
\text { (Cognitive } \\
\text { flexibility) }\end{array}$ & $\begin{array}{l}\text { Habilidad para cambiar a nuevas } \\
\text { actividades, hacer frente a cambios en } \\
\text { las rutinas, aprender de los errores y } \\
\text { elaborar estrategias alternativas, }\end{array}$ & $\begin{array}{l}\text { El instrumento de evaluación utilizado fue NEUROPSI: } \\
\text { Evaluación Neuropsicológica Breve en Español (Ostrosky } \\
\text { Solís et al., 2012). } \\
\text { La batería Neuropsi evalúa la atención, la memoria y las } \\
\text { funciones ejecutivas a través de } 13 \text { subescalas. Estas subescalas }\end{array}$ \\
\hline
\end{tabular}


se agrupan en cinco secciones: I. Orientación, II. Atención y Concentración, III. Funciones Ejecutivas, IV. Memoria Codificación, y V. Memoria Evocación.

La suma de las puntuaciones directas permite obtener tres índices globales de ejecución en a) Atención-Funciones Ejecutivas, b) Memoria y c) Total Atención y Memoria. Las puntuaciones directas se transforman en puntuaciones normalizadas clasificándose la ejecución de cada sujeto según los rangos que se muestran en la Tabla 2.

Tabla 2.

Clasificación según la puntuación normalizada

\begin{tabular}{ccc}
\hline Puntuación total normalizada & Clasificación \\
\hline 116 & 127 & Normal alto \\
85 & 115 & Normal \\
70 & 84 & Alteración leve \\
0 & 69 & Alteración severa \\
\hline
\end{tabular}

Los datos analizados en este trabajo corresponden al índice de Atención-Funciones Ejecutivas, ya que son los que permiten establecer el perfil de los sujetos.

La verificación de la adecuación del lenguaje de las pruebas se realizó mediante una entrevista a cinco docentes de las unidades educativas involucradas en el estudio. También se seleccionó, de forma aleatoria, a 10 estudiantes para conocer el nivel de educación y comprensión que poseen los estudiantes. Esta verificación permitió determinar que las preguntas no requerían modificación alguna.

\subsection{Análisis estadístico}

Debido a la situación sanitaria mundial, la aplicación de la prueba se realizó, individualmente, a través de la plataforma virtual de video conferencia Zoom.

Para conocer los datos perdidos, errores y valores atípicos se realizó el análisis exploratorio de datos. El proceso de consolidación de información se realizó en una hoja de cálculo Excel para verificar la información proporcionada; y obtener datos de acuerdo con el interés de la investigación.

\subsection{Aspectos éticos}

Los consentimientos informados fueron aplicados de forma individual a los padres o tutores de cada menor. Se les dio a conocer el propósito del estudio, el manejo seguro y confidencial de los datos, así como el uso con fines académicos.

\section{Resultados}

Los resultados obtenidos se evaluaron en función de la puntuación global obtenida por los estudiantes, el sexo y los años de escolarización.

\subsection{Puntuación global del índice Atención-Función Ejecutiva}

El análisis de las puntuaciones totales de los 135 menores nos ha permitido constatar que la mayoría de ellos, el 93,33\%, obtienen resultados que se enmarcan en una ejecución "normal". En la Tabla 3 se puede ver que solo un estudiante obtuvo un resultado normal alta, y que 2 presentaban una alteración severa.

La Figura 1 presenta la distribución de los menores en función de la puntuación global obtenida en Atención-Función Ejecutiva.

Tabla 3.

Distribución de los menores en función de su puntuación global

\begin{tabular}{|c|c|c|c|c|}
\hline \multicolumn{2}{|c|}{$\begin{array}{l}\text { Criterio de } \\
\text { evaluación } \\
\mathrm{P} \text { máxima- } \mathrm{P} . \\
\text { mínima }\end{array}$} & \multirow{2}{*}{$\begin{array}{l}\text { Clasificación } \\
\text { Normal alto }\end{array}$} & \multirow{2}{*}{$\begin{array}{c}\text { Número de } \\
\text { Estudiantes } \\
1\end{array}$} & \multirow{2}{*}{$\begin{array}{c}\% \\
0,74 \%\end{array}$} \\
\hline 116 & 127 & & & \\
\hline 85 & 115 & Normal & 126 & $93,33 \%$ \\
\hline 70 & 84 & Alt. leve & 6 & $4,44 \%$ \\
\hline 0 & 69 & Alt. severa & 2 & $1,48 \%$ \\
\hline \multicolumn{2}{|c|}{ Total } & & 135 & $100 \%$ \\
\hline
\end{tabular}

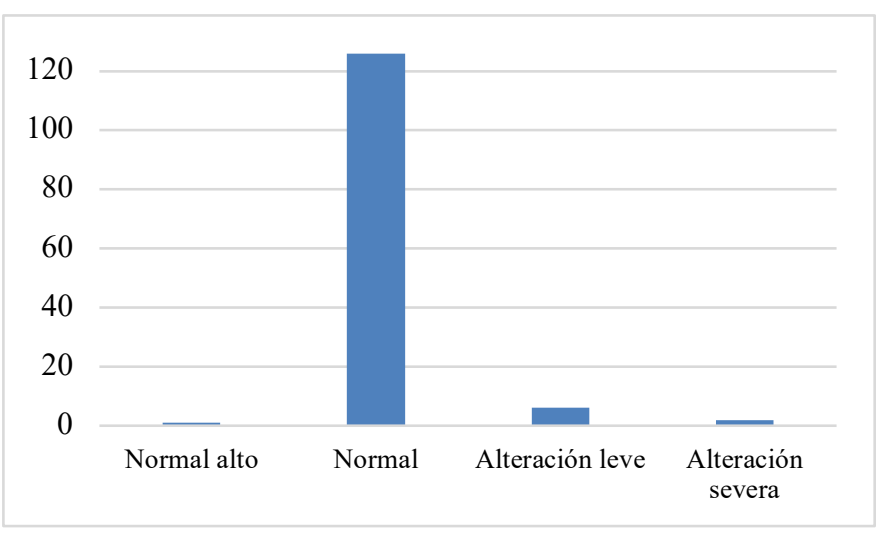

Figura 1. Distribución de los menores en función de su puntuación global

\subsection{Resultados en función del sexo}

Los resultados globales en función del sexo de los sujetos se muestran en la Tabla 4. Como se puede observar la distribución de los criterios de clasificación son semejantes para los hombres y las mujeres (Figura 2).

Tabla 4.

Resultados según el sexo.

\begin{tabular}{|c|c|c|c|c|c|c|}
\hline \multirow{2}{*}{\multicolumn{2}{|c|}{$\begin{array}{c}\text { Criterio de } \\
\text { evaluación } \\
\text { Propuesto atención } \\
\text { y funciones } \\
\text { ejecutivas }\end{array}$}} & \multirow[b]{2}{*}{$\begin{array}{l}\text { Clasifica- } \\
\text { ción }\end{array}$} & \multicolumn{4}{|c|}{ Sexo } \\
\hline & & & M & $\%$ & $\mathrm{~F}$ & $\%$ \\
\hline 116 & 127 & $\begin{array}{l}\text { Normal } \\
\text { alto }\end{array}$ & 1 & $\begin{array}{c}0,74 \\
\%\end{array}$ & 0 & $\begin{array}{c}0,00 \\
\%\end{array}$ \\
\hline
\end{tabular}




\begin{tabular}{|c|c|c|c|c|c|c|}
\hline 85 & 115 & Normal & 66 & $\begin{array}{c}48,89 \\
\% \\
\end{array}$ & $\begin{array}{l}6 \\
0 \\
\end{array}$ & $\begin{array}{c}44,4 \\
4 \% \\
\end{array}$ \\
\hline 70 & 84 & $\begin{array}{c}\text { Alteración } \\
\text { leve }\end{array}$ & 3 & $\begin{array}{c}2,22 \\
\%\end{array}$ & 3 & $\begin{array}{c}2,22 \\
\%\end{array}$ \\
\hline 0 & 69 & $\begin{array}{c}\text { Alteración } \\
\text { severa }\end{array}$ & 1 & $\begin{array}{c}0,74 \\
\%\end{array}$ & 1 & $\begin{array}{c}0,74 \\
\%\end{array}$ \\
\hline \multicolumn{3}{|c|}{ TOTAL } & 71 & $\begin{array}{c}52,59 \\
\%\end{array}$ & $\begin{array}{l}6 \\
4\end{array}$ & $\begin{array}{c}47,4 \\
1 \%\end{array}$ \\
\hline
\end{tabular}

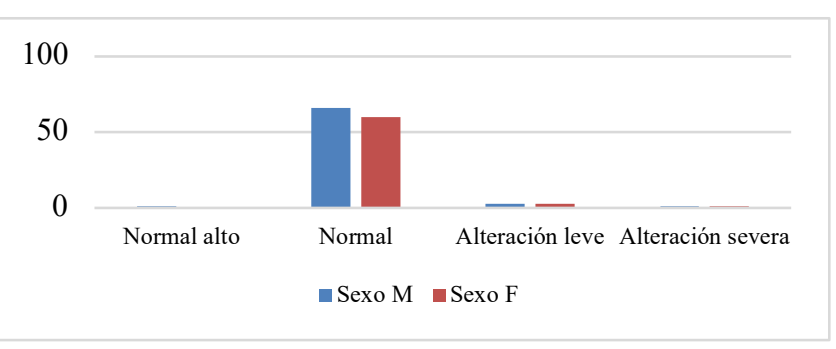

Figura 2. Distribución de los resultados según el sexo

\subsection{Resultados en función de los años de escolaridad}

Como se puede observar en la Tabla 6 , los sujetos con más años de escolaridad presentan un mayor número de resultados clasificados normales. En los grupos con cinco y siete años de escolaridad, existe un menor con la clasificación de alteración severa, y en el de siete años, uno con clasificación normal alto

Tabla 5.

Resultado de los menores en función de los años de escolaridad

\begin{tabular}{|c|c|c|c|c|c|c|c|c|}
\hline \multirow{2}{*}{\multicolumn{2}{|c|}{$\begin{array}{c}\text { Criterio de } \\
\text { evaluación } \\
\text { Propuesto atención } \\
\text { y funciones } \\
\text { ejecutivas } \\
\text { Puntuación total } \\
\text { normalizada }\end{array}$}} & \multirow{3}{*}{$\begin{array}{l}\text { Clasifi- } \\
\text { cación } \\
\\
\begin{array}{c}\text { Normal } \\
\text { alto }\end{array}\end{array}$} & \multicolumn{6}{|c|}{ Años de escolaridad } \\
\hline & & & 5 & $\%$ & 6 & $\%$ & 7 & $\%$ \\
\hline 116 & 127 & & 0 & $\begin{array}{c}0,00 \\
\%\end{array}$ & 0 & $\begin{array}{c}0,00 \\
\%\end{array}$ & 1 & $\begin{array}{c}1,68 \\
\%\end{array}$ \\
\hline 85 & 115 & Normal & $\begin{array}{l}3 \\
2 \\
\end{array}$ & $\begin{array}{c}88,8 \\
9 \% \\
\end{array}$ & $\begin{array}{l}3 \\
7 \\
\end{array}$ & $\begin{array}{c}92,5 \\
\%\end{array}$ & $\begin{array}{l}5 \\
7 \\
\end{array}$ & $\begin{array}{c}96,6 \\
1 \% \\
\end{array}$ \\
\hline 70 & 84 & $\begin{array}{l}\text { Alteraci } \\
\text { ón leve }\end{array}$ & 3 & $\begin{array}{c}8,33 \\
\%\end{array}$ & 3 & $\begin{array}{l}7,5 \\
\%\end{array}$ & 0 & $\begin{array}{c}0,00 \\
\%\end{array}$ \\
\hline 0 & 69 & $\begin{array}{l}\text { Alteraci } \\
\text { ón } \\
\text { severa }\end{array}$ & 1 & $\begin{array}{c}2,78 \\
\%\end{array}$ & 0 & $\begin{array}{c}0,00 \\
\%\end{array}$ & 1 & $\begin{array}{c}1,68 \\
\%\end{array}$ \\
\hline & & & \multicolumn{6}{|c|}{ Años de escolaridad } \\
\hline \multicolumn{3}{|c|}{ Total } & 36 & $\begin{array}{c}26,6 \\
7 \%\end{array}$ & 40 & $\begin{array}{c}29,6 \\
3 \%\end{array}$ & 59 & $\begin{array}{c}43,7 \\
0 \%\end{array}$ \\
\hline
\end{tabular}

\section{CONCLUSIONES}

La evaluación de los alumnos del Cantón de Ambato nos permite afirmar que la mayoría de ellos obtienen resultados "normales" respecto a sus grupos normativos.

También se ha podido observar que no existen en la distribución de las puntuaciones en función del sexo, y que las puntuaciones más altas y las más bajas pertenecen, en su mayoría a los menores con mayor edad.

El análisis de cada una de las subpruebas que componen el índice Atención-Función Ejecutiva permitirá profundizar en las diferencias intragrupos y en el perfil de cada uno de los sujetos participantes.

Los datos analizados nos han permitido definir diferentes grupos de estudio en los que aplicar la batería de tareas diseñadas. La evaluación de dicho programa nos permitirá establecer líneas de trabajo con los estudiantes de primaria, con el objetivo de mejorar su rendimiento en FE.

\section{AgRADECIMIENTOS}

Los autores agradecen a la Universidad Técnica de Ambato (UTA) y a la Dirección de Investigación y Desarrollo (DIDE) por el apoyo brindado para la ejecución exitosa de este trabajo a través del proyecto de investigación titulado "Potenciación de funciones ejecutivas a través de un programa de entrenamiento cerebral y aprendizaje mediado", y a la Universidad Complutense de Madrid por su colaboración. Al PhD Paúl Santiago Pullas Tapia quien fue coordinador inicial del proyecto de investigación e impulsó con su liderazgo inigualable la creación y desarrollo del mismo, para que al momento esté consolidado y desde el cielo lo guíe.

\section{REFERENCIAS}

Anderson, P. (2002). Assessment and development of executive function (EF) during childhood. Child Neuropsychology, $8,71-82$.

Anderson, P. J. (2008). Towards a developmental model of executive function. En V. Anderson, R. Jacobs y P. J. Anderson (Eds.), Executive functions and the frontal /lobes: A lifespan perspective (pp. 3-22). Nueva York: Psychology Press.

Anderson, V. y Spencer-Smith, M. (2013). Children's frontal lobes: No longer silent. In D. Stuss, y R. Knight (Eds.), Principles of frontal lobe function (2 ed., pp. 118134). Oxford University Press.

Barkley, R. A. (1997). ADHD and the nature of self-control. New York: Guilford.

Barkley, R. A. (2001). The inattentive type of ADHD as a distinct disorder: What remains to be done. Clinical Psychology: Science and Practice, 8(4), 489-501.

Bronowski, J. (1990). Science and Human Value. New York: Harper Colophon Books

Curtis, W.J., Lindeke, L.L., Georgieff, M.K. y Nelson C.A. (2002). Neurobehavioral functioning in neonatal intensive care unit graduates in late childhood and early adolescence, Brain, 125, 1646-1659. 
Colombo B. (2020). Brain and Art: From Aesthetics to Therapeutics. Switzerland: Springer International Publishing.

Damasio, A. R. (1996). El error de Descartes. Barcelona: Crítica.

Fuster, J. (1997). The Prefrontal Cortex Anatomy, Physiology and Neuropsychology of the Frontal Lobe. Filadelfia: Lippincott-Raven.

García-Molina A, Enseñat-Cant allops A, Tirapu-Ustárroz J, Roig-Rovira T. (2009). Maduración de la corteza prefrontal y desarrollo de las funciones ejecutivas durante los primeros cinco años de vida. Revista de Neurología, 48: 435-40.

Gilbert, S.J. y Burgess, P.W. (2008). Executive function. Current Biology, 18: R110-4.

Goldman-Rakic P.S. (1995). Cellular basis of working memory. Neuron 14 (3): 447-485.

Lezak, M.D. (1982). The problem of assessing executive functions. International Journal of Psychology, 17: 28197.

Lezak, M. D. (1995). Neuropsychological assessment (3rd ed.). New York: Oxford University Press.
Miyake A, Friedman N.P., Emerson M.J., Witzki A.H., Howerter A. y Wager T.D. (2000). The unity and diversity of executive functions and their contributions to complex "frontal lobe" tasks: a latent variable analysis. Cognitive Psychoogyl. 41:49-100.

Ostrosky Solís, F., Gómez M., Matute, E., Rosselli, M., Ardila, A. y Pineda, D. (2012). Neuropsi: Atención y Memoria. Manual. Méjico: Manual Moderno.

Pino Melgarejo, M., y Urrego Betancourt, Y. (2013). La importancia de las funciones ejecutivas para el desarrollo de las competencias ciudadanas en el contexto educativo. Cultura Educación y Sociedad, 4(1).

Soprano, A.M. (2003). Evaluación de las funciones ejecutivas en el niño. Revista de Neurología, 37(01).

Tirapu-Ustárroz J, Cordero-Andrés P, Luna-Lario P, HernáezGoñi P. (2017). Propuesta de un modelo de funciones ejecutivas basado en análisis factoriales. Revista de Neurología, 64: 75-84.

Verdejo-García A, Bechara A. (2010). Neuropsicología de las funciones ejecutivas. Psicothema, 22: 227-35.

Zelazo, P. D., Frye, D., y Rapus, T. (1996). An age-related dissociation between knowing rules and using them. Cognitive Development, 11(1), 37-63. 